\title{
Transesophageal echocardiographic evaluation of positioning of central venous catheter determined by Peres formula and radiologic landmark based technique
}

\author{
Il Seok Kim, \\ Kangdong Sacred Heart Hospital, Hallym University Medical Center, \\ Dept of Anesthesiology \& Pain Medicine, Seoul, Korea, Republic of
}

\begin{abstract}
Background
- Central venous catheterization is a standard clinical practice for infusion of fluids, blood products, or vasoactive drugs to the central circulation and to monitor central venous pressure during perioperative periods.

- This procedure can lead to significant complications, including cardiac arrhythmia, vascular injury, hydrothorax, hemothorax, hydromediastinum, and cardiac tamponade. Such complications can occur when the catheter tip is inserted within the heart chamber or when it abuts the vascular wall at a steep angle.

- Although ideal positioning of the catheter tip is controversial according to short-term or long-term use and catheter performance, the lower SVC near the RA/SVC junction might be the optimal position of the catheter tip for preventing malfunction, migration, and thrombosis.
\end{abstract}

\section{Objective}

- Peres formula relating body height to catheter insertion depth has been widely used in traditional landmark-based central venous catheterization.

- We determined the catheter insertion depth with a new formula using the sternoclavicular joint and the carina as landmarks, with $1.5 \mathrm{~cm}$ safety margin.

- The accuracy of tip positioning with radiologic landmark based technique $(\mathrm{R})$ and Peres formula $(\mathrm{P})$ was compared in this study using TEE.

\section{Methods}

- Real-time ultrasound guided central venous catheterization was done through the right internal jugular or subclavian vein.

- Patients were randomly assigned to either the P group ( $\mathrm{n}=93$ ) or the $\mathrm{R}$ group (n=95).

- In the P group, catheter insertion depth was derived from the Peres formula as height $/ 10 \mathrm{~cm}$ for the right IJV and (height/10) $-2 \mathrm{~cm}$ for the right SCV.

- In the $\mathrm{R}$ group, the vertical length from the midpoint of the right sternoclavicular joint to the carina was measured from the preoperative chest PA using an internal measuring tool available on PACS. Catheter insertion depth was determined by surface and radiological landmarks, and was estimated by adding a distance from the skin insertion point to the right sternoclavicular joint, the vertical length from the right sternoclavicular joint to the carina, and an additional $1.5 \mathrm{~cm}$.

- In both groups, after the guidewire was advanced and secured at the determined depth, the bicaval view of TEE identified the location of the j-tip in relationship to the RA/SVC junction (Fig. 1). The RA/SVC junction on TEE was determined as the base of the superior edge of the crista terminalis.

- The area within $2 \mathrm{~cm}$ above and $1 \mathrm{~cm}$ below the RA/SVC junction was considered as the target zone.

- The catheter was passed over the inserted guidewire and secured at the determined depth. The catheter position within the lumen of the SVC was examined to determine whether the catheter was located in the center of the lumen or abutted the medial or lateral wall.

- The angle of the catheter tip to the vascular wall was measured.

- Normal saline $2 \mathrm{ml}$ was used as an echographic contrast and was flushed through the catheter. Microbubbles on the distal tip were monitored to determine whether the flow stream of microbubbles hit the vascular wall or not (Fig. 2).
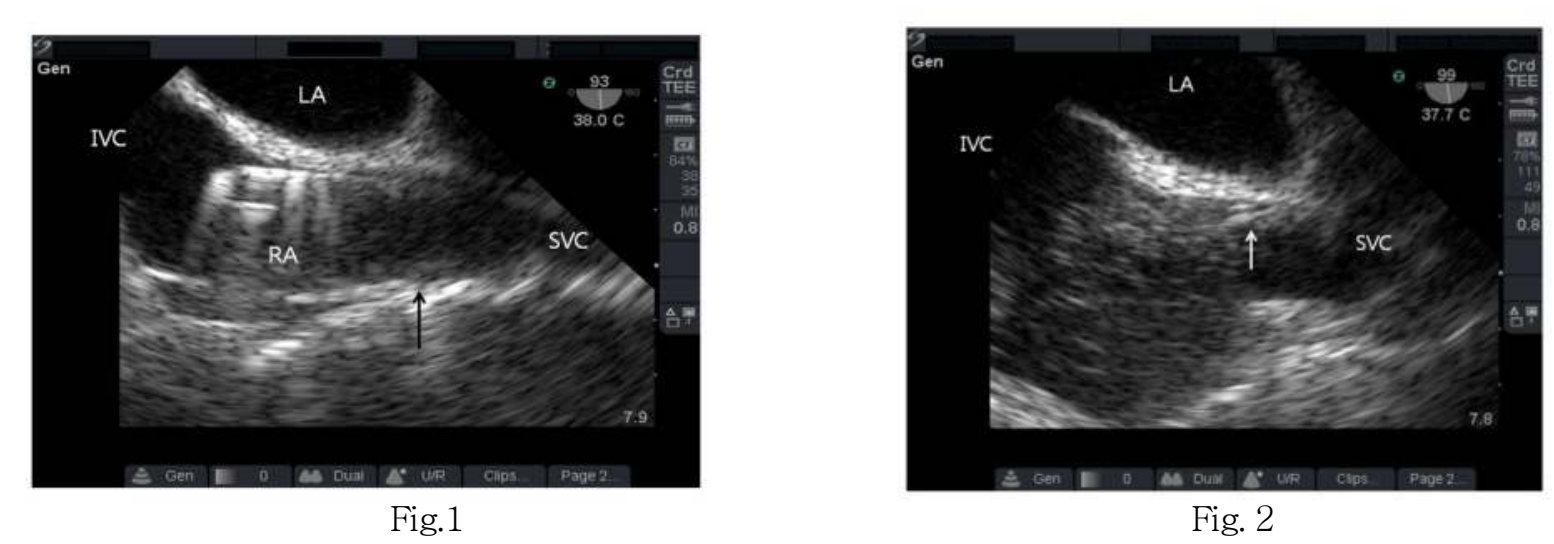

Results

- Distance from skin insertion point to the RA/SVC junction and determined depth of catheter insertion were more strongly correlated in the $\mathrm{R}$ group (17.4 [1.2] $\mathrm{cm}$ and 16.7 [1.5] $\mathrm{cm}, \mathrm{r}=0.821, \mathrm{P}<0.001)$ than in the $\mathrm{P}$ group $(17.3[1.2] \mathrm{cm}$ and $16.4[1.1] \mathrm{cm}, \mathrm{r}=0.517, \mathrm{P}<0.001)$, with $\mathrm{z}=3.96(\mathrm{P}<0.001)$.

- More tips were correctly positioned in the $\mathrm{R}$ group than in the $\mathrm{P}$ group $(74.2 \%$ vs $92.6 \%, \mathrm{P}=0.001)$.

- Abutment, tip angle to the lateral wall $>40^{\circ}$, and disrupted flow stream were comparable between the groups.

- There were no complications including haemothorax, hydrothorax, pneumothorax, and cardiac tamponade, in either group.
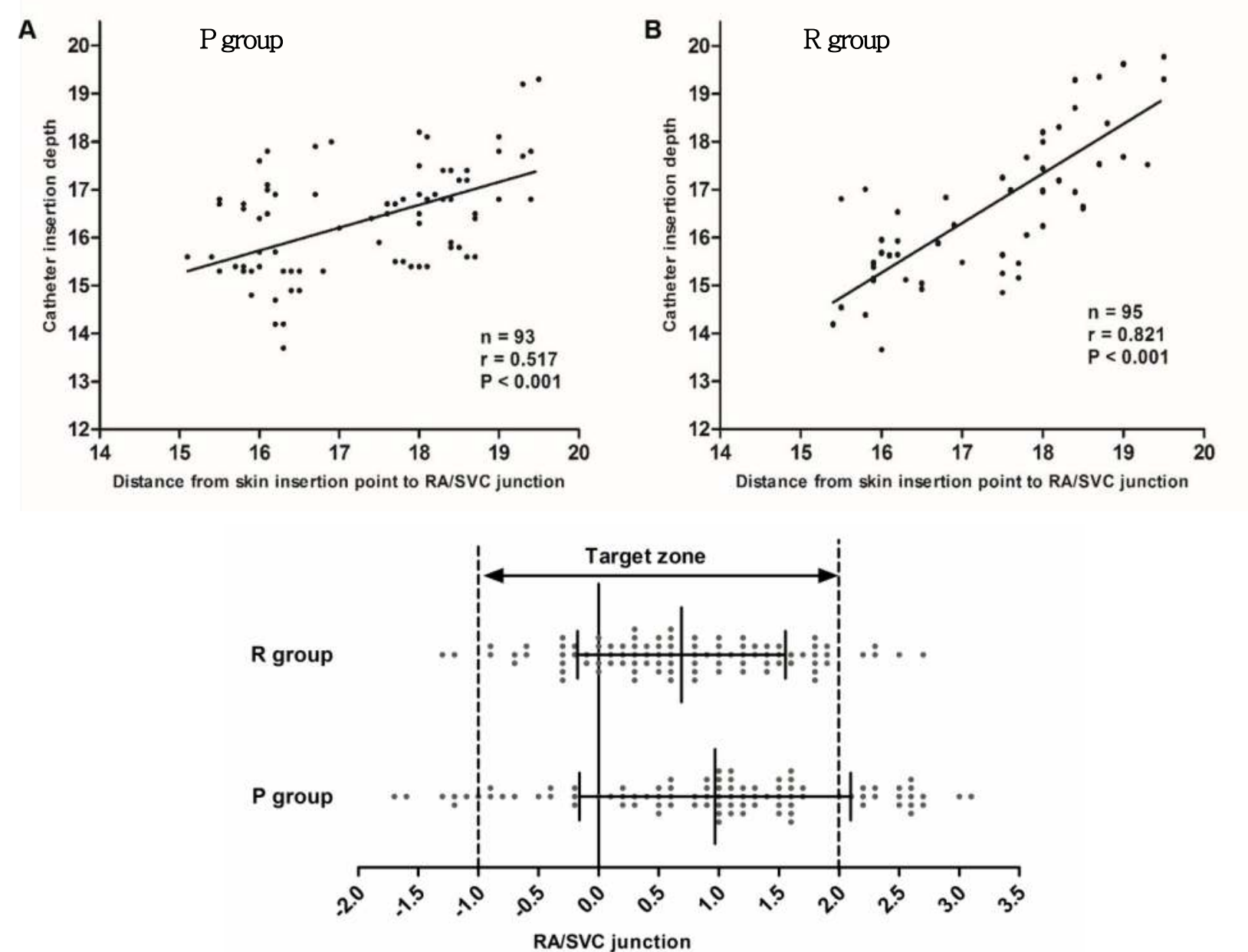

\begin{tabular}{lccc}
\hline Location & $\begin{array}{c}\text { Peres formula group } \\
{[\mathrm{n}(\%)](\mathrm{n}=93)}\end{array}$ & $\begin{array}{c}\text { Radiological landmark group } \\
{[\mathrm{n}(\%)](\mathrm{n}=95)}\end{array}$ & P value \\
\hline Optimal position & $69(74)$ & $88(93)$ & $0.001^{*}$ \\
Position below target zone & $6(6.5)$ & $2(2.1)$ & 0.132 \\
Position above target zone & $18(19)$ & $5(5.3)$ & $0.003^{*}$ \\
Abutment to vascular wall & $2(2.2)$ & $3(3.2)$ & 0.510 \\
Angle of tip $\left(>40^{\circ}\right)$ & 0 & 0 & - \\
Flow stream hitting vascular wall & $3(3.2)$ & $1(1.1)$ & 0.303 \\
\hline
\end{tabular}

\section{Conclusion}

- This radiological landmark-based technique was more accurate than the Peres formula to locate the catheter tip in the lower SVC near the $\mathrm{RA} / \mathrm{SVC}$ junction, for real-time ultrasound-guided right-side central venous catheterization.

- If the catheter tip is placed within the center of lumen, allowing free movement without impingement on the vessel wall and guaranteeing adequate flow, the possibility of cardiac tamponade would be unlikely, even if the catheter tip is located below the pericardial reflection. 